\title{
Improved Algorithmic Results for Unsplittable Stable Allocation Problems
}

\author{
Ágnes Cseh ${ }^{1}$ and Brian C. Dean ${ }^{2}$ \\ 1 Institute for Mathematics, TU Berlin \\ 2 School of Computing, Clemson University
}

\begin{abstract}
The stable allocation problem is a many-to-many generalization of the well-known stable marriage problem, where we seek a bipartite assignment between, say, jobs (of varying sizes) and machines (of varying capacities) that is "stable" based on a set of underlying preference lists submitted by the jobs and machines. Building on the initial work of [6], we study a natural "unsplittable" variant of this problem, where each assigned job must be fully assigned to a single machine. Such unsplittable bipartite assignment problems generally tend to be NP-hard, including previously-proposed variants of the unsplittable stable allocation problem [13. Our main result is to show that under an alternative model of stability, the unsplittable stable allocation problem becomes solvable in polynomial time; although this model is less likely to admit feasible solutions than the model proposed in [13, we show that in the event there is no feasible solution, our approach computes a solution of minimal total congestion (overfilling of all machines collectively beyond their capacities). We also describe a technique for rounding the solution of a stable allocation problem to produce "relaxed" unsplit solutions that are only mildly infeasible, where each machine is overcongested by at most a single job.
\end{abstract}

\section{Introduction}

Consider a bipartite assignment problem over a graph $G=(V=J \cup M, E)$ involving the assignment of a set of jobs $J$ to a set of machines $M$. Each job $j \in J$ has a processing time $q(j)$, each machine $m \in M$ has a capacity $q(m)$, and there is a capacity $c(j m)$ for each edge $j m \in E$ governing the maximum amount of job $j$ that can be assigned to machine $m$. A feasible assignment of jobs to machines is described by a function $x: E \rightarrow \mathbb{R}_{\geq 0}$ such that

1. $0 \leq x(j m) \leq c(j m)$ for all edges $j m \in E$,

2. $x(j):=\sum_{m \in M} x(j m) \leq q(j)$ for all jobs $j \in J$, and

3. $x(m):=\sum_{j \in J} x(j m) \leq q(m)$ for all machines $m \in M$.

If $x(j m) \in\{0, q(j)\}$ for all $j m \in E$, we say the assignment is unsplit, since each assigned job is assigned in its entirety to a single machine. We often forgo the use of edge capacities $c(j m)$ when discussing unsplit assignments, since an edge $j m$ can simply be deleted if $c(j m)<q(j)$. 
Problems of the form above have been extensively studied in the algorithmic literature, where typical objectives are to find a feasible assignment or one of maximum weight (maximizing a linear objective function $\sum_{j m \in E} w(j m) x(j m)$, where $w(j m)$ is the weight of edge $j m \in E$ ). While the fractional (splittable) variants of these problems are easy to solve in polynomial time via network flow techniques, the unsplittable variants are somewhat more interesting. In linear time, one can greedily assign jobs arbitrarily to machines until no further assignments are possible, thereby producing a maximal assignment. However, if we care about finding an unsplit assignment of measurably good quality, the problem becomes substantially harder. It is NP-hard to find an unsplit assignment of either maximum total size $|x|=\sum_{j m \in E} x(j m)$ or of maximum weight; the former is a variant of the multiple subset sum problem [4, and the latter is known as the multiple knapsack problem [5].

In contrast to problems with explicit edge costs, the stable allocation problem is an "ordinal" problem variant where the quality of an assignment is expressed in a more game theoretic setting via ranked preference lists submitted by the jobs and machines, with respect to which we seek an assignment that is stable (defined shortly). In this paper, we study the stable allocation problem in the unsplittable setting, which was shown to be NP-hard in [13] using one natural definition for stability. We show here that by contrast, a different and more strict notion of stability, proposed initially in [6], leads to an $O(|E|)$ algorithm for the unsplit problem. The tradeoff is that under this different notion of stability, it is unlikely that feasible solutions will exist. However, we show that by relaxing the problem to allow mildly infeasible solutions, our algorithm computes a "relaxed" unsplit stable solution (in which each machine is filled beyond its capacity by at most the allocation of a single job) in which the total amount of overcongestion across all machines, $\sum_{m \in M} \max (0, x(m)-q(m))$, is minimized (so in particular, if there is a feasible solution with no congestion, we will find it).

Through the work of several former authors 818]17], the "relaxed" model has become relatively popular in the context of unsplittable bipartite assignment and unsplittable flow problems. The standard approximation algorithm framework (finding an approximately-optimal, feasible solution) typically does not fit these problems, since finding any feasible solution is typically NP-hard. Instead, authors tend to focus on pseudo-approximation results with minimal congestion per machine or per edge. Analogous results were previously developed for unsplit stable allocation problems in [6], where an unsplit stable allocation can be found in linear time in which each machine is overcongested by at most a single job. The model of stability proposed in [6] is the one we further develop in this paper, and among all of these prior approaches (including those for standard unsplittable bipartite assignment and flows), it seems to be the only unsplit model studied to date in which minimization of total congestion is possible in polynomial time. Hence, there is a substantial algorithmic incentive to consider this model, even though its notion of stability is less natural than in [13].

In our "relaxed" unsplit model, we develop new structural and algorithmic results by showing how to compute in $O(|E|)$ time a "job-optimal" assignment 
that maximizes the total size $|x|$ of all assigned jobs, and a "machine-optimal" assignment that minimizes $|x|$. It is this machine-optimal solution that we show also minimizes total congestion. In order to produce potentially other solutions (e.g., that might be more fair to both sides), we show also a technique for "rounding" a solution of the fractional stable allocation problem to obtain a relaxed unsplit solution. Finally, we comment on several mathematical properties of the set of all relaxed unsplit solutions, showing that while they unfortunately seem to lack the nice distributed lattice structure satisfied by solutions of the stable matching and allocation problems, they do at least adhere to a weakened form of the so-called "rural hospital" theorem, defined shortly.

\section{Background and Preliminaries}

\subsection{Stable Matching and Allocation Problems}

Stable Marriage. The stable marriage (or stable matching) problem takes place on a bipartite graph with men on one side and women on the other, where each individual submits a strictly-ordered, but possibly incomplete preference list of the members of the opposite sex. The goal is to find a matching that is stable, containing no blocking pair - an unmatched (man, woman) pair $(m, w)$ where $m$ is either unmatched or prefers $w$ to his current partner, and likewise for $w$.

In their seminal paper [10, Gale and Shapley describe a simple $O(|E|)$ algorithm to find a stable matching for any instance. The most typical incarnation of their algorithm generates a solution that is "man-optimal" and "womanpessimal", where each man is matched with the best possible partner he could receive in any stable matching, and each woman is matched with the worst possible partner she could receive in any stable matching. By reversing the roles of the men and women, the algorithm can also generate a solution that is simultaneously woman-optimal and man-pessimal.

Stable Allocation. The stable allocation problem was introduced by Baïou and Balinski [1] as a high-multiplicity variant of the stable matching problem, where we match non-unit elements with non-unit elements - here, we speak of matching jobs of varying size with machines of varying capacity. Just as before, jobs and machines submit strict preferences over their outgoing edges in the bipartite assignment graph. If job $j \in J$ prefers machine $m_{1} \in M$ to machine $m_{2} \in M$, we write $\operatorname{rank}_{j}\left(j m_{1}\right)>\operatorname{rank}_{j}\left(j m_{2}\right)$. A stable allocation in this setting is a feasible allocation (as defined in the introduction) where for every edge $j m \in E$ with $x(j m)<c(j m)$, either $j$ is fully assigned to machines at least as good as $m$, or $m$ is fully assigned to jobs at least as good as $j$. That is, there can be no blocking edge $j m$ where $x(j m)<c(j m)$ and both $j$ and $m$ would prefer use more of this edge. For sake of simplicity, we say that edges with positive $x$ value are in $x$. Machines with $x(m)=q(m)$ are saturated. Later, when $x(m)>q(m)$ occurs in the relaxed version of the problem, we talk about over-capacitated machines. If any job prefers machine $m$ to any of its allocated machines, then $m$ is called popular, otherwise $m$ is unpopular. Note that all popular machines must be saturated in any stable allocation. 
The stable allocation problem can be solved in $O(|E| \log |V|)$ time [7]. There can be many different solutions for the same instance, but they all have the same total allocation $|x|$, and even stronger, the values of $x(j)$ and $x(m)$ for each job and machine remain unchanged across all stable allocations. This holds for both stable marriage and stable allocation, moreover, even for stable roommate, the non-bipartite version of the problem, and is known as the rural hospital theorem. A common application of stable matching in practice is the National Resident Matching Program (NRMP), where medical school graduates in the USA are matched with residency positions at hospitals via a centralized stable matching procedure. A consequence of the rural hospital theorem is that if a less-preferred (typically rural) hospital cannot fill its quota in some stable assignment, then there is no stable assignment in which its quota will be filled.

Like the stable marriage problem, one can always find job-optimal, machinepessimal and job-pessimal, machine-optimal solutions. To define these notions for the stable allocation problem, Baïou and Balinski 1] define an order on stable solutions based on a min-min criterion, where a job $j$ prefers allocation $x_{1}$ to allocation $x_{2}$ if $x_{1}(j m)<x_{2}(j m)$ implies $x_{1}\left(j m^{\prime}\right)=0$ for every $j m^{\prime}$ worse than $j m$ for $j$. A similar relation can be defined for machines as well. Stable matchings 12 and stable allocations 1 ] both form distributive lattices with an ordering relation based on the min-min criterion.

\subsection{Unsplittable Stable Allocation Problems}

An unsplit allocation $x$ satisfies $x(j m) \in\{0, q(j)\}$ for all $j m \in E$, so each job is assigned in its entirety to one machine. For simplicity, we introduce a "dummy" machine $m_{d}$ with high capacity, which acts as the last choice for every job. This lets us assume without loss of generality that an unsplittable assignment always exists in which every job is assigned. In this context, we define the size $|x|$ of an assignment so that jobs assigned to $m_{d}$ do not count, since they are in reality unassigned. In addition to the application of scheduling jobs in a non-preemptive fashion, a motivating application for the unsplittable stable allocation problem is in assigning personnel with "two-body" constraints. For example, in the NRMP, a married pair of medical school graduates might act as an unsplittable entity of size 2 (this particular application has been studied in substantial detail in the literature $20314 \mid 15]$ ).

From an algorithmic standpoint, one of the main results of this paper is that how we define stability in the unsplit case seems quite important. In [13, the following natural definition was proposed: an edge $j m$ is blocking if $j$ prefers $m$ to its current partner, and if $m$ prefers $j$ over $q(j)$ units of its current allocation. Unfortunately, it was shown in 13 that this definition makes the computation of an unsplit stable assignment NP-hard. We therefore consider an alternate, stricter notion of stability where edge $j m$ is blocking if $j$ prefers $m$ to its current partner, and if $m$ prefers $j$ over any amount of its current allocation. That is, if $j$ would prefer to be assigned to $m$ over its current partner, than $m$ must be saturated with jobs $m$ prefers to $j$. As in the splittable case, popular machines must therefore be saturated. Practice shows [16] that if a hospital is willing to 


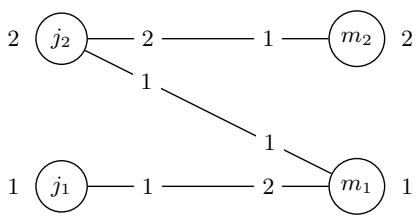

Fig. 1. This instance admits an unsplit assignment, but the unique stable allocation is fractional.

hire one person in a couple, but it has no free job opening for the partner, it is most likely amenable to make room for both applicants. Therefore, our definition of a blocking pair serves practical purposes.

The existence of an unsplit stable allocation cannot be guaranteed. A simple instance where the unique stable allocation is fractional is shown in Figure 1. The quota of each job and machine is displayed next to the vertex, while the preference lists are displayed on the edges.

Relaxed Unsplit Assignments. The downside of our alternate definition of stability is that it is unlikely to allow feasible unsplit stable allocations to exist in most large instances. Therefore, we consider allowing mildly-infeasible solutions where each machine can be over-capacitated by a single job - a model popularized by previous results in the approximation algorithm literature for standard unsplittable assignment problems 818 17, and introduced in the context of unsplittable stable allocation by Dean et al. [6. Specifically, we say $x$ is a relaxed unsplit assignment if $x(j m) \in\{0, q(j)\}$ for every edge $j m \in E$, and if for each machine $m$, removal of the least-preferred job assigned to $m$ would cause $x(m)<q(m) \sqrt{3}$. Our definition of stability extends easily naturally to the relaxed setting: we say a relaxed unsplit assignment $x$ is stable if for every edge $j m$ with $x(j m)=0$, either $j$ is assigned to a machine $j$ prefers to $m$, or $m$ 's quota is filled or exceeded with jobs $m$ prefers to $j$. Otherwise, if edge $j m$ with $x(j m)=0$ is preferred by $j$ to its allocated machine and $m$ 's quota is not filled up with better edges than $j m$, then $j m$ blocks $x$.

Note that the relaxed unsplit model differs from the non-relaxed unsplit model with capacities inflated by $\max q(j)$, since stability is still defined with respect to the original capacities. It may be best to regard "capacities" in this setting as constraints governing start time, rather than completion time of jobs, since a machine below its capacity is always willing to launch a new job, irrespective of job size. Similarly, a machine $m$ views an edge $j m$ as blocking if the machine is not fully saturated with jobs $m$ prefers to $j m$, as in this case $m$ is

${ }^{3}$ The model introduced in [6] allows $x(m) \leq q(m)$, but we believe strict inequality is actually a better choice - for mathematical reasons as well as from a modeling standpoint. For example, the old definition applied to a hospital-resident matching scenario with married couples might cause a hospital to accept two more residents than its quota, while the new definition would only require accepting one more resident. All of the results in [6] hold with either definition. 
willing to accept $\mathrm{jm}$. The "capacity" of a machine therefore reflects the cutoff at which it feels content to receive additional assignment versus when it can no longer accept additional load.

\section{Machine-Optimal Relaxed Unsplit Assignments}

In [6], a version of the Gale-Shapley algorithm is described to find the job-optimal relaxed unsplit stable assignment $x_{\text {jopt }}$. In this context, job-optimal means that there is no relaxed unsplit stable assignment $x^{\prime}$ such that any job is assigned to a better machine in $x^{\prime}$ than in $x_{\text {jopt }}$. The implementation described in [6] runs in $O(|E| \log |V|)$ time, but $O(|E|)$ is also easy to achieve. In this section, we show how to define and compute a machine-optimal relaxed unsplit stable allocation $x_{\text {mopt }}$ also in $O(|E|)$ time, and we prove the following:

Theorem 1. Among all relaxed unsplit stable allocations $x,|x|$ is maximized at $x=x_{\text {jopt }}$ and minimized at $x=x_{\text {mopt }}$.

One of the main challenges with computing a machine-optimal assignment is defining machine-optimality. In the stable allocation problem, existence of a machine-optimal solution follows from the fact that all stable solutions form a distributive lattice under the standard min-min ordering relationship introduced in 11. However, this ordering seems to depend crucially on the existence of a rural hospital theorem, which no longer holds in the relaxed unsplit case, since relaxed unsplit stable assignments may differ in cardinality, as shown in the upper-left example in Figure 2 The dashed edges form a stable solution of size 3, while the remaining edges build another stable solution of size 6. Even an appropriately relaxed version of the rural hospital theorem seems difficult to formulate over relaxed instances: machines can be saturated or even over-capacitated in one relaxed unsplit stable solution, while being empty in another one. The lowerleft example in the figure shows such an instance: the two stable assignments are denoted with the same line types, and $m_{1}$ is the machine that has different positions in them. Nonetheless, we can still prove a result in the spirit of the rural hospital theorem, which we discuss further in Section 3.3.

Without an "exact" rural hospital theorem, comparing two allocations using the original min-min ordering seems problematic, and indeed one can construct instances where two relaxed unsplit stable solutions are incomparable according to this criterion. For example, the instance on the right in Figure 2 shows two relaxed unsplit solutions (indicated with dotted and solid edges) that are incomparable for machine $m_{3}$. We therefore adopt a different but nonetheless natural ordering relation: lexicographical order. We say that machine $m$ prefers unsplit allocation $x_{1}$ to allocation $x_{2}$ if the best edge in $x_{1} \triangle x_{2}$ belongs to $x_{1}$, where $\triangle$ denotes the symmetric difference operation. The opposite ordering relation is based on the position of jobs, and since jobs are always assigned to machines in an unsplit fashion, the lexicographic and min-min relations are actually the same from the job's perspectives; hence, "job optimal" means the same thing under both. The lexicographical position of the same agent in different allocations can 


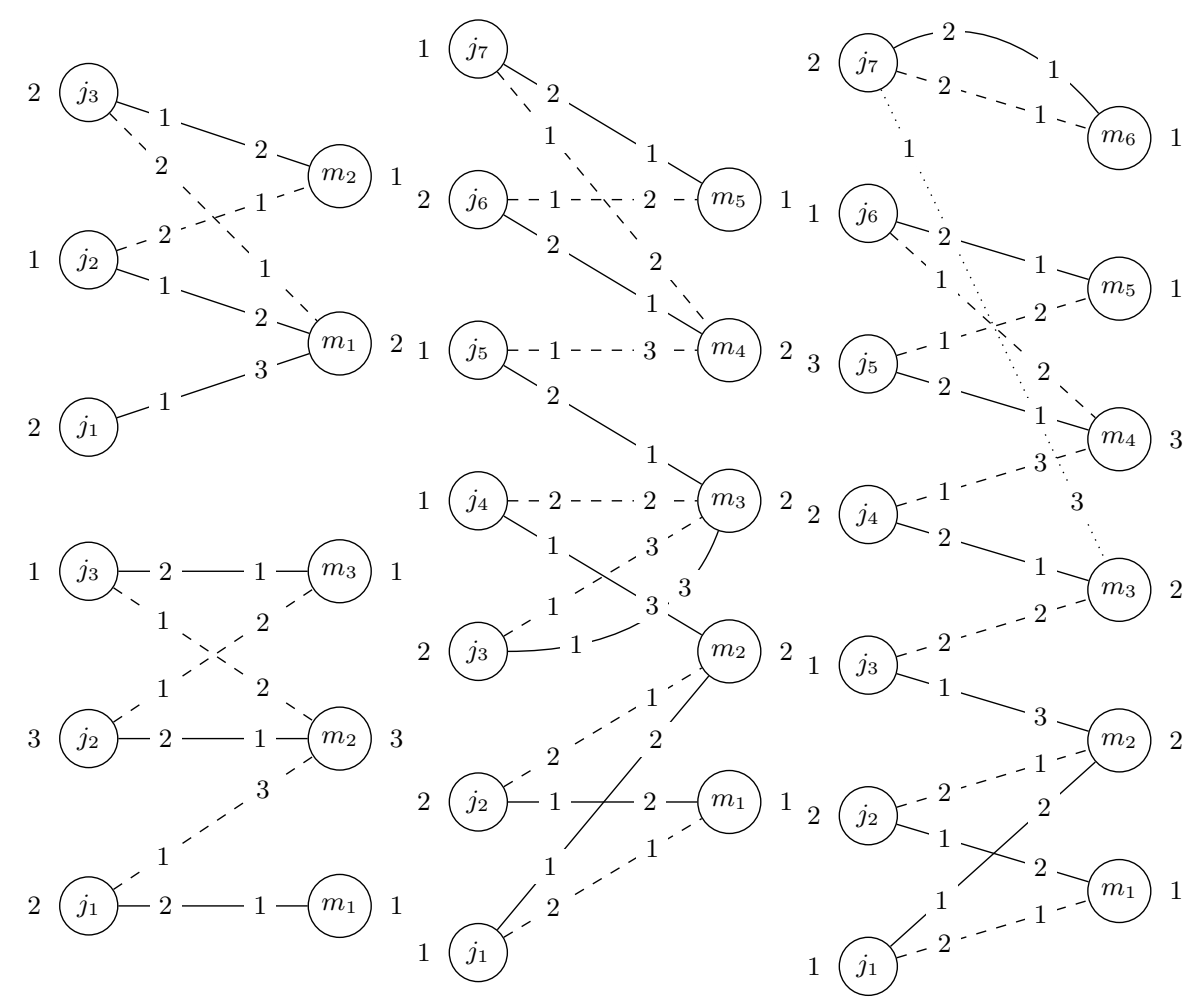

Fig. 2. The upper-left instance admits two relaxed unsplit solutions differing in cardinality. The lower-left example is evidence against a rural hospital theorem. The graph in the middle shows two incomparable relaxed unsplit solutions. The last instance is a counterexample showing the difficulty of formulating join and meet operations. The first and third graphs illustrate instances of NRMP.

always be compared, and we say a relaxed stable solution $x$ is machine-optimal if it is at least as good for all machines as any other relaxed stable assignment (although we still need to show that such a solution always exists).

\subsection{The Reversed Gale-Shapley Algorithm}

For the classical stable marriage problem, the Gale-Shapley algorithm can be reversed easily, with women proposing instead of men, to obtain a woman-optimal solution. We show that this idea can be generalized (carefully accounting for multiple assignment and congestion among machines) to compute a machineoptimal relaxed unsplit stable assignment. Pseudocode for the algorithm appears in Figure 3 . 
1: $x\left(j m_{d}\right):=q(j)$ for all $j \in J, x(j m):=0$ for every other $j m \in E$

2: while $\exists m: x(m)<q(m)$ with a non-empty preference list do

$m$ proposes to its best job $j$ with $q(j)$

if $j$ prefers $m$ to its current partner then

$x(j m):=q(j)$

$x\left(j m^{\prime}\right):=0$ for $\forall m^{\prime} \neq m$

end if

delete $j$ from $m$ 's preference list

9: end while

Fig. 3. Reversed relaxed unsplit Gale-Shapley algorithm.

Claim. The algorithm terminates in $O(|E|)$ time.

Proof. In each step, a job is deleted from a machine's preference list.

Claim. The algorithm produces an allocation $x$ that is a relaxed unsplit stable assignment.

Proof. First, we check the three feasibility constraints for $x$. Since proposals are always made with $q(j)$ and refusals are always full rejections, the quota constraints of the jobs may not be violated. Moreover, each job is assigned to exactly one machine. Machines can be over-capacitated, but deleting the worst job from their preference list results in an allocation under their quota. Otherwise the machine would not have proposed along the last edge.

If $x$ is unstable, then there is an empty edge $j m$ blocking $x$. During the execution, $m$ must have proposed to $j$. This offer was rejected, because $j$ already had a better partner in the current allocation. Since jobs monotonically improve their position in the assignment, this leads to a contradiction.

Claim. The output $x$ is the machine-optimal relaxed unsplit stable assignment. That is, no machine has a better lexicographical position in any other relaxed unsplit stable assignment than in $x$.

Proof. Assume that there is a relaxed unsplit stable assignment $x^{\prime}$, where some machines come better off than in $x$. To be more precise, in the symmetric difference $x \triangle x^{\prime}$, the best edge incident to these machines belongs to $x^{\prime}$. When running the reversed relaxed unsplit Gale-Shapley algorithm, there is a step when the first such edge $j m_{1}$ carries a proposal from $m_{1}$ but gets rejected. Otherwise, $m_{1}$ filled up or exceeded its quota in $x$ with only better edges than $j m_{1}$. Let us consider only this edge first and denote the feasible, but possibly unstable relaxed allocation produced by the algorithm so far by $x_{0}$.

When $j$ refused $j m_{1}$, it already had a partner $m_{0}$ in $x_{0}$, better than $m_{1}$. Even if there is no guarantee that $j m_{0} \in x$, it is sure that $j m_{0} \notin x^{\prime}$ and $j m_{0}$ does not block $x^{\prime}$, though $\operatorname{rank}_{j}\left(j m_{0}\right)>\operatorname{rank}_{j}\left(j m_{1}\right)$ for $j m_{1} \in x^{\prime}$. It is only possible if $m_{0}$ is saturated or over-capacitated in $x^{\prime}$ with edges better than $j m_{0}$. Since $j m_{0} \in x_{0}, x_{0}$ may not contain all of these edges, otherwise $m_{0}$ is congested 
in $x_{0}$ beyond the level required for a relaxed unsplit assignment. During the execution of the reversed relaxed unsplit Gale-Shapley algorithm, $m_{0}$ proposed along all of these edges and got rejected by at least one of them. This edge is never considered again, it may not enter $x$ later. Thus, $j m_{1}$ is not the first edge in $x^{\prime} \backslash x$ that was rejected in the algorithm.

With this, we completed the constructive proof of the following theorem:

Theorem 2. The machine-optimal relaxed unsplit stable assignment $x_{m o p t}$ can be computed in $O(|E|)$ time.

\subsection{Properties of the Job- and Machine-Optimal Solutions}

Theorem 3. The job-optimal relaxed unsplit stable assignment $x_{j o p t}$ is the machinepessimal relaxed unsplit stable assignment and vice versa, the machine-optimal relaxed unsplit stable assignment $x_{m o p t}$ is the job-pessimal relaxed unsplit stable assignment.

Proof. We start with the first statement. Suppose that there is a relaxed unsplit stable assignment $x^{\prime}$ that is worse for some machine $m$ than $x_{\text {jopt }}$. This is only possible if $m$ 's best edge $j m$ in $x_{\text {jopt }} \triangle x^{\prime}$ belongs to $x_{\text {jopt }}$. Since $x_{\text {jopt }}$ is the joboptimal solution, $j m^{\prime}, j^{\prime}$ 's edge in $x^{\prime}$ is worse than $j m$. But then, $m$ is saturated or over-capacitated in $x^{\prime}$ with better edges than $j m$. We assumed that all edges in $x^{\prime}$ that are better than $j m$ are also in $x_{\text {jopt }}$. Thus, omitting $m$ 's worst job from $x_{\text {jopt }}$ leaves $m$ at or over its quota.

The second half of the theorem can be proved similarly, using the reversed Gale-Shapley algorithm. Assume that there is a relaxed unsplit stable assignment $x^{\prime}$ that assigns some jobs to worse machines than $x_{\text {mopt }}$ does. Let us denote the set of edges preferred by any job to its allocated machine in $x^{\prime}$ by $E^{\prime}$. Due to our indirect assumption, $E^{\prime}$ contains some edges of $x_{\text {mopt }}$. When running the reversed Gale-Shapley algorithm on the instance, there is an edge $j m \in E^{\prime}$ that is the first edge in $E^{\prime}$ carrying a proposal. Since $j$ is not yet matched to a better machine, it also accepts this offer. Even if $j m \notin x_{\text {mopt }}, j$ 's edge in $x_{\text {mopt }}$ is at least as good as $m$, because jobs always improve their position during the course of the reversed Gale-Shapley algorithm. On the other hand, $m$ cannot fulfill its quota in $x_{\text {mopt }}$ with better edges than $j m$, simply because the proposal step along $j m$ took place.

Since $j m \notin x^{\prime}$, but $j$ prefers $j m$ to its edge in $x^{\prime}, m$ is saturated or overcapacitated with better edges than $j m$ in $x^{\prime}$. As observed above, not all of these edges belong to $x_{\text {mopt }}$. Let us denote one of them in $x^{\prime} \backslash x_{\text {mopt }}$ by $j^{\prime} m$. Before proposing along $j m, m$ submitted an offer to $j^{\prime}$ that has been refused. The only reason for such a refusal is that $j^{\prime}$ has already been matched to a better machine $m^{\prime}$. But since $j^{\prime} m \in x^{\prime}, j^{\prime} m^{\prime} \in E^{\prime}$. This contradicts to our indirect assumption that $j m$ is the first edge in $E^{\prime}$ that carries a proposal.

Theorem 1 also follows from the proof above. 
We note that although we can compute the job-optimal and machine-optimal relaxed unsplit stable allocations, there in general does not appear to be an obvious underlying lattice structure behind relaxed unsplit solutions. As already mentioned above, stable matchings and fractional stable allocations form a distributive lattice. In those cases, computing the meet or join of two solutions is fairly easy. In order to reach the join of $x_{1}$ and $x_{2}$, all machines choose the better edge set out of those two allocations 9 . Similarly, for meet, jobs are allowed to chose. The example in Figure ?? illustrates that this property does not carry over to relaxed unsplit assignments. If all jobs chose the better allocation, $m_{3}$ remains empty and $j_{7} m_{3}$ becomes blocking. Similar examples can easily be constructed to show that choosing the worse assignment also can lead to instability.

Our ability to compute $x_{\text {mopt }}$ in $O(|E|)$ time now gives us a linear-time method for solving the (non-relaxed) unsplittable stable allocation problem (according to our stricter notion of stability).

Lemma 1. If an instance $\mathcal{I}$ admits an unsplit stable assignment $x$, then the machine-optimal relaxed unsplit stable assignment $x_{\text {mopt }}$ on the corresponding relaxed instance $\mathcal{I}^{\prime}$ is also an unsplit stable assignment on $\mathcal{I}$.

Proof. Suppose the statement is false, e.g. although there is an unsplit stable assignment $x, x_{\text {mopt }}$ is no unsplit stable assignment on $\mathcal{I}$. This can be due to two reasons: either the feasibility or the stability of $x_{\text {mopt }}$ is harmed on $\mathcal{I}$. The latter case is easier to handle. An allocation that is feasible on both instances and stable on $\mathcal{I}^{\prime}$ may not be blocked by any edge on $\mathcal{I}$, since the set of unsaturated edges is identical on both instances. The second case, namely if $x_{\text {mopt }}$ violates some feasibility constraint on $\mathcal{I}$, needs more care.

$\mathcal{I}$ and $\mathcal{I}^{\prime}$ differ only in the constraints on the quota of machines. If $x_{\text {mopt }}$ is infeasible on $\mathcal{I}$, then there is a machine $m$ for which $x_{\text {mopt }}\left(m_{1}\right)>q\left(m_{1}\right)$. Regarding the unsplit stable assignment $x$, the inequality $x\left(m_{1}\right) \leq q\left(m_{1}\right)$ trivially holds. Now we use Theorem 1 for $x$ and $x_{\text {mopt }}$ that are both relaxed unsplit stable assignments on $\mathcal{I}^{\prime}$. This corollary implies that if there is a machine $m_{1}$ with $x_{\text {mopt }}\left(m_{1}\right)>x\left(m_{1}\right)$, then another machine $m_{2}$ exists for which $x_{\text {mopt }}\left(m_{2}\right)<x\left(m_{2}\right)$ holds.

This machine $m_{2}$ plays a crucial role in our proof. It has a lower allocation value in the machine-optimal relaxed solution $x_{\text {mopt }}$ than in another relaxed stable solution $x$ on $\mathcal{I}$. Its lexicographical position can only be better in $x_{\text {mopt }}$ than in $x$ if the best edge $j_{2} m_{2}$ in $x \triangle x_{\text {mopt }}$ belongs to $x_{\text {mopt }}$. Moreover, $x \triangle x_{\text {mopt }}$ also contains an edge $j_{3} m_{2} \in x$, otherwise $x_{\text {mopt }}\left(m_{2}\right)>x\left(m_{2}\right)$. Naturally, $\operatorname{rank}_{m}\left(j_{2} m_{2}\right)<\operatorname{rank}_{m}\left(j_{3} m_{2}\right)$. At this point, we use the property that $x_{\text {mopt }}\left(m_{2}\right)<q\left(m_{2}\right)$. Since $m_{2}$ has free quota in $x_{\text {mopt }}$ and $j_{3} m_{2}$ is not a blocking edge, $j_{3}$ must be matched to a machine better than $m_{2}$ in $x_{\text {mopt }}$. Thus, there is a job that comes better off in the machine-optimal (and job-pessimal) relaxed solution than in another relaxed stable solution. This contradiction to Theorem 3 finishes our proof.

Lemma 1 shows that if there is an unsplit solution, it can be found in linear time by computing the machine-optimal relaxed solution. Unfortunately, the 
existence of such an unsplit assignment is not guaranteed. Our next result applies to the case when no feasible unsplit solution can be found. In terms of congestion, with the machine-optimal solution we come as close as possible to feasibility.

Theorem 4. Amongst all relaxed unsplit stable solutions, $x_{\text {mopt }}$ has the lowest total congestion.

Proof. Let $M_{u}$ denote the set of machines that remain under their quota in $x_{\text {mopt }}$. Note that $\sum_{m \notin M_{u}} x_{\text {mopt }}(m)$, the total allocation value on the remaining machines clearly determines $\sum_{m \notin M_{u}} x_{\text {mopt }}(m)-q(m)$, the total congestion of $x_{\text {mopt }}$. Let $x$ be an arbitrary relaxed solution. Due to Theorem 1 the total allocation value is minimized at $x_{\text {mopt }}$. Therefore, for any relaxed unsplit stable allocation $x$, the following inequalities hold:

$$
\begin{aligned}
\sum_{m \in M} x(m) & \geq \sum_{m \in M} x_{\text {mopt }}(m) \\
\sum_{m \notin M_{u}} x(m)+\sum_{m \in M_{u}} x(m) & \geq \sum_{m \notin M_{u}} x_{\text {mopt }}(m)+\sum_{m \in M_{u}} x_{\text {mopt }}(m) \\
\sum_{m \notin M_{u}} x(m)-\sum_{m \notin M_{u}} x_{\text {mopt }}(m) & \geq \sum_{m \in M_{u}} x_{\text {mopt }}(m)-\sum_{m \in M_{u}} x(m) \\
\sum_{m \notin M_{u}}(x(m)-q(m))-\sum_{m \notin M_{u}}\left(x_{\text {mopt }}(m)-q(m)\right) & \geq \sum_{m \in M_{u}} x_{\text {mopt }}(m)-\sum_{m \in M_{u}} x(m)
\end{aligned}
$$

At this point, we investigate the sign of both sides of the last inequality. The core of our proof is to show that for each $m \in M_{u}$ and relaxed stable solution $x$, $x_{\text {mopt }}(m) \geq x(m)$. This result, proved below, has two benefits. On one hand, the term on the right hand-side of the last inequality is non-negative. Therefore, the inequality implies that the total congestion on machines in $M \backslash M_{u}$ is minimized at $x_{\text {mopt }}$. On the other hand, no machine in $M_{u}$ is over-capacitated in any relaxed solution. Thus, the total congestion is minimized at $x_{\text {mopt }}$.

Lemma 2. For every $m \in M_{u}$ and relaxed solution $x$, the inequality $x_{m o p t}(m) \geq$ $x(m)$ holds.

Proof. Suppose that there is a machine $m \in M_{u}$ for which $x_{\text {mopt }}(m)<x(m)$ for some relaxed solution $x$. Since $m$ is unsaturated in $x_{\text {mopt }}$, it is unpopular. On the other hand, there is at least one job $j$ for which $j m \in x \backslash x_{\text {mopt }}$. As $m$ is unpopular in $x_{\text {mopt }}, j$ is allocated to a better machine in $x_{\text {mopt }}$ than in $x$. Since $x_{\text {mopt }}$ is the job-pessimal solution, we derived a contradiction.

\subsection{A Variant of the "Rural Hospital" Theorem}

In the relaxed unsplit case, we have provided counterexamples against an exact rural hospital theorem (e.g., where all machines have the same amount of allocation in all relaxed unsplit allocations) or even a weakened theorem stating that all unsaturated / congested machines have the same status in all relaxed unsplit 
allocations. The examples in Figure 2 also show that no similar property holds for the jobs' side either. Lemma 2 above however suggests an alternate variant of "rural hospital" theorem that does hold.

Theorem 5. A machine $m$ that is not saturated in $x_{\text {mopt }}$ will not be saturated in every relaxed unsplit stable solution, and a machine $m$ that is over-capacitated in $x_{\text {jopt }}$ must at least be saturated in every relaxed unsplit stable solution.

Proof. The first part is shown by Lemma 2, For the second part, consider a machine $m$ that is over-capacitated in $x_{\text {jopt }}$ but has $x(m)<q(m)$ in some relaxed unsplit allocation $x$. Consider any job $j$ in $x_{\text {jopt }} \backslash x$, and note that since $x_{\text {jopt }}$ is job-optimal, $j$ prefers $m$ to its partner in $x$. Hence, $j m$ blocks $x$.

As of the jobs' side, Theorem 3 already guarantees that if a job is unmatched in $x_{\text {jopt }}$, then it is unmatched in all relaxed stable solutions and similarly, if it is matched in $x_{\text {mopt }}$, then it is matched in all relaxed stable solutions.

\section{Rounding Algorithms}

We have seen now how to compute $x_{\text {jopt }}$ and $x_{\text {mopt }}$ in linear time. We now describe how to find potentially other relaxed unsplit solutions by "rounding" solutions to the (fractional) stable allocation problem. For example, this could provide a heuristic for generating relaxed unsplit solutions that are more balanced in terms of fairness between the jobs and machines. Our approach is based on augmentation around rotations, alternating cycles that are commonly used in stable matching and allocation problems to move between different stable solutions (see, e.g., 7[11]).

We begin with a stable allocation $x$ with $x(j)=q(j)$ for every job $j$, thanks to the existence of a dummy machine. For each job $j$ that is not fully assigned to its first-choice machine, we define its refusal edge $r(j)$ to be the worst edge $j m$ incident to $j$ with $x(j m)>0$. Jobs with refusal edges also have proposal edges - namely all their edges ranked better than $r(j)$. Recall that a machine with incoming proposal edges is said to be popular. We call a machine dangerous if it is over-capacitated and has zero assignment on all its incoming proposal edges.

Claim. Consider a popular machine $m$ in some fractional stable allocation $x$. Amongst all proposal edges incoming to $m$, at most one has positive allocation value in $x$, and this positive proposal edge is ranked lower on $m$ 's preference list than any other edge into $m$ with positive allocation.

Proof. Let $\operatorname{rank}_{m}\left(j_{1} m\right)>\operatorname{rank}_{m}\left(j_{2} m\right)$ be proposal edges such that $x\left(j_{1} m\right)$ and $x\left(j_{2} m\right)$ are both positive. Note that $j_{1} m$ blocks $x$, since $j_{1}$ and $m$ have worse allocated edges in $x$. A similar argument implies the last part of the claim.

Our algorithm proceeds by a series of augmentations around rotations, defined as follows. We start from a popular, non-dangerous machine $m$ (if no such machine exists, the algorithm terminates, having reached an unsplit solution). 
Since $m$ is popular and non-dangerous, it has incoming proposal edges with positive allocation, and due to the preceding claim, it must have exactly one such edge $j m$. We include $j m$ as well as $j$ 's refusal edge $j m^{\prime}$ in our partial rotation, then continue building the rotation from $m^{\prime}$ (again finding an incoming proposal edge, etc.). We continue until we close a cycle, visiting some machine $m$ visited earlier (in which case we keep just the cycle as our rotation, not the edges leading up to the cycle), or until we reach a machine $m$ that is unpopular or dangerous, where our rotation ends.

To enact a rotation, we increase the allocation on its proposal edges by $\varepsilon$ and decrease along the refusal edges by $\varepsilon$, where $\varepsilon$ is chosen to be as large as possible until either (i) a refusal edge along the rotation reaches zero allocation, or (ii) a dangerous machine at the end of the rotation drops down to being exactly saturated from being over-capacitated, and hence ceases to be dangerous. We call case (i) a "regular" augmentation. This concludes the algorithm description.

Claim. The algorithm terminates after $O(|E|)$ augmentations.

Proof. Jobs remain fully allocated during the whole procedure, and their lexicographical positions never worsen. With every regular augmentation, some edge stops being a refusal edge, and will never again be increased or serve as a proposal or refusal edge. We can therefore have at most $O(|E|)$ regular augmentations. Furthermore, a machine can only become dangerous if one of its incoming refusal pointers reaches zero allocation, so the number of newly-created dangerous machines over the entire algorithm is bounded by $|E|$. Hence, the number of non-regular augmentations is at most $O(|M|+|E|)=O(|E|)$.

Claim. The final allocation $x$ is a feasible relaxed unsplit assignment.

Proof. Since we start with a feasible assignment and jobs never lose or gain allocation, the quota condition on jobs cannot be harmed. If there is any edge $j m$ with $0<x(j m)<q(j)$, then $j$ has at least two positive edges, the better one must be a positive proposal edge. This contradicts the termination condition, and hence $x$ is unsplit.

We now show that deleting the worst job from each machine results in an allocation strictly below the machine's quota. It is clearly true at the beginning, where no machine is over-capacitated (since $x$ starts out as a feasible stable allocation). The only case when $x(m)$ increases is when $m$ is the first machine on a rotation. As such, $m$ has a positive proposal edge $j m$, which is also its worst allocated edge, due to our earlier claim.

- If $m$ is not over-capacitated when choosing the rotation, then even if $x(j m)$ rises as high as $q(j)$, this increases $x(m)$ by strictly less than $q(j)$. Thus, deleting $j m$, the worst allocated edge of $m$, guarantees that $x(m)$ sinks under $q(m)$.

- If $m$ is saturated or over-capacitated when choosing the rotation, then $j m$ would have been the best proposal edge of $m$ earlier, when $x(m)$ was not greater than $q(m)$. Thus, assigning $j$ entirely to $m$ does not harm the relaxed 
quota condition. Let us consider the last step as $x(m)$ exceeded $q(m)$. Again, $m$ was the starting vertex of an augmenting path, having a positive proposal edge. If it was $j m$, our claim is proved. Otherwise $m$ became over-capacitated while $x(j m)$ was zero, and then increased the allocation on $j m$. But between those two operations, $m$ had to become dangerous, because it switched its best proposal edge to $j m$. Dangerous machines never start alternating paths. Thus, we have a contradiction to the fact that we considered the last step when $x(m)$ exceeded $q(m)$.

Claim. The final allocation $x$ is stable.

Proof. Suppose some edges block $x$. Since we started with a stable allocation, there was a step during the execution of the algorithm when the first edge $j m$ became blocking. Before this step, either $j$ or $m$ was saturated or overcapacitated with better edges than $\mathrm{jm}$. The change can be due to two reasons:

1. $j$ gained allocation on an edge worse than $j m$, or

2. $m$ gained allocation on an edge worse than $j m$.

As already mentioned, $j$ 's lexicographical position never worsens: $\operatorname{rank}_{j}(p)>$ $\operatorname{rank}_{j}(r(j))$ always holds. The second event also may not occur, because machines always play their best response strategy. An edge $j m$ that becomes blocking when allocation is increased on an edge worse than it, was already a proposal edge before. Thus, $m$ would have chosen $j m$, or an edge better than $j m$ to add it to the augmenting path.

Since each augmentation requires $O(|V|)$ time and there are $O(|E|)$ augmentations, our rounding algorithm runs in $O(|E||V|)$ total time. If desired, dynamic tree data structures can be used (much like in [7]) to augment in $O(\log |V|)$ time, bringing the total time down to just $O(|E| \log |V|)$.

Although jobs improve their lexicographical position in each rotation, the output of the algorithm is not necessarily $x_{\text {jopt }}$. In fact, even $x_{\text {mopt }}$ can be reached via this approach. Ideally, this approach can serve as a heuristic to generate many other relaxed unsplit stable allocations, if run from a variety of different initial stable solutions $x$.

\section{References}

1. Mourad Baïou and Michel Balinski. The stable allocation (or ordinal transportation) problem. Math. Oper. Res., 27(3):485-503, 2002.

2. Péter Biró, Robert W. Irving, and Ildikó Schlotter. Stable matching with couples: An empirical study. J. Exp. Algorithmics, 16:1.2:1.1-1.2:1.27, May 2011.

3. Péter Biró and Flip Klijn. Matching with couples: A multidisciplinary survey. International Game Theory Review (IGTR), 15(02):1340008-1-1, 2013.

4. Alberto Caprara, Hans Kellerer, and Ulrich Pferschy. A $\{$ PTAS $\}$ for the multiple subset sum problem with different knapsack capacities. Information Processing Letters, 73(3-4):111 - 118, 2000. 
5. Chandra Chekuri and Sanjeev Khanna. A polynomial time approximation scheme for the multiple knapsack problem. SIAM J. Comput., 35(3):713-728, 2005.

6. Brian C. Dean, Michel X. Goemans, and Nicole Immorlica. The unsplittable stable marriage problem. In Gonzalo Navarro, Leopoldo E. Bertossi, and Yoshiharu Kohayakawa, editors, IFIP TCS, volume 209 of IFIP, pages 65-75. Springer, 2006.

7. Brian C. Dean and Siddharth Munshi. Faster algorithms for stable allocation problems. Algorithmica, 58(1):59-81, 2010.

8. Y. Dinitz, N. Garg, and M.X. Goemans. On the single-source unsplittable flow problem. Combinatorica, 19:17-41, 1999.

9. Tamás Fleiner. On stable matchings and flows. In Proceedings of the 36th International Workshop on Graph-Theoretic Concepts in Computer Science, WG'10, pages 51-62, Berlin, Heidelberg, 2010. Springer-Verlag.

10. David Gale and Lloyd Shapley. College admissions and the stability of marriage. American Mathematical Monthly, 1:9-14, 1962.

11. D. Gusfield and R.W. Irving. The Stable Marriage Problem: Structure and Algorithms. MIT Press, 1989.

12. Donald E. Knuth. Mariages stables et leurs relations avec d'autres problèmes combinatoires. Collection de la Chaire Aisenstadt. Les Presses de l'Université de Montréal, Montréal, Québec, Canada, 1976. Edition revue et corrigée, 1981. Currently available from Les Publications CRM / Centre de Recherches Mathématiques, Université de Montréal, Montréal, Québec.

13. Eric McDermid and David Manlove. Keeping partners together: algorithmic results for the hospitals/residents problem with couples. J. Comb. Optim., 19(3):279-303, 2010.

14. Eytan Ronn. NP-complete stable matching problems. J. Algorithms, 11(2):285304, 1990.

15. Alvin E. Roth. The evolution of the labor market for medical interns and residents: A case study in game theory. Journal of Political Economy, 92:991-1016, 1984.

16. Alvin E. Roth. The national residency matching program as a labor market. Journal of the American Medical Association, 275(13):1054-1056, 1996.

17. D.B. Shmoys and É. Tardos. Scheduling unrelated machines with costs. In Proceedings of the 4 th annual ACM-SIAM Symposium on Discrete algorithms (SODA), pages 448-454, 1993.

18. M. Skutella. Approximating the single source unsplittable min-cost flow problem. In Proceedings of the 41st Annual Symposium on Foundations of Computer Science (FOCS), pages 136-145, 2000. 